\title{
Global weighted regularity estimates for higher order elliptic and parabolic systems
}

The Quan Bui ${ }^{1}$ and Xuan Truong Le$^{2}$

${ }^{1}$ Dong Nai University

${ }^{2}$ University of Economics Ho Chi Minh City, Ho Chi Minh City, Viet Nam

August 12, 2020

\begin{abstract}
In this paper, we prove the weighted Lorentz and weighted Orlicz estimates for the weak solutions to the higher order parabolic systems with the leading coefficients satisfying a small BMO norm condition. As a byproduct, we obtain the weighted estimates for the higher order elliptic systems.
\end{abstract}

Hosted file

WeightedRegularityEstimatesForHigherOrderEllipticAndParapolic-submitted.pdf available at https://authorea.com/users/350494/articles/475318-global-weighted-regularity-estimatesfor-higher-order-elliptic-and-parabolic-systems 